GEOMETRY IN NONLINEAR CONTROL

AND DIFFERENTIAL INCLUSIONS

BANACH CENTER PUBLICATIONS, VOLUME 32

INSTITUTE OF MATHEMATICS

POLISH ACADEMY OF SCIENCES

WARSZAWA 1995

\title{
STABILITY OF NONAUTONOMOUS SYSTEMS BY LIAPUNOV'S DIRECT METHOD
}

\author{
DIRK AEYELS \\ Department of Systems Dynamics, University of Gent \\ Technologiepark-Zwijnaarde, 9, 9052 Gent (Zwijnaarde), Belgium \\ E-mail: Dirk.Aeyels@rug.ac.be
}

\begin{abstract}
This paper discusses asymptotic stability for nonautonomous systems by means of the direct method of Liapunov. The existence of a positive time-invariant Liapunov function with negative semi-definite derivative is assumed. The paper focuses on the extra conditions needed in order to guarantee asymptotic stability. The proposed criterion is compared with the standard results available for autonomous systems. A specialization and extension is obtained for a class of linear nonautonomous systems.
\end{abstract}

1. Introduction. The classical Liapunov approach to the study of asymptotic stability of an equilibrium of autonomous differential equations relies on the existence of a positive definite Liapunov function with negative definite time derivative. There exist however large classes of differential equations and associated natural continuously differentiable Liapunov functions $V(x)$ which exhibit negative semidefinite time derivatives. It was pointed out by Barbashin and Krasovskii [3, 4] that for these cases asymptotic stability is still guaranteed when $\{x: \dot{V}(x)=0\}$ does not contain nontrivial solutions.

This result also follows from LaSalle's invariance principle which itself is based on the invariance of the limit sets of solutions of autonomous differential equations: it says that for some $V$ with $\dot{V} \leq 0$, bounded solutions converge to the largest invariant set contained in $\{x: \dot{V}(x)=0\}$. The result of Barbashin and Krasovskii on asymptotic stability of the origin follows when $V(0)=0$ and $V(x)>0$ outside 0 , i.e. when $\mathrm{V}$ is positive definite.

1991 Mathematics Subject Classification: Primary 34D05.

This paper presents research results of the Belgian Programme on Interuniversity Poles of Attraction initiated by the Belgian State, Prime Minister's Office for Science, Technology and Culture. The author gratefully acknowledges additional research support from the EC-Science Project SC1-0433-C(A). The scientific responsibilty rests with the author.

The paper is in final form and no version of it will be published elsewhere. 
For nonautonomous systems the situation is more complex. Some of the results above are no longer true. This is due to the lack of invariance of the limit sets with respect to the flow. The existence of a positive definite Liapunov function $V(x)$ with negative definite $\dot{V}(x, t)$ still implies asymptotic stability [8]. When a negative semidefinite $\dot{V}$ is allowed, LaSalle's principle collapses in general for nonautonomous systems, although e.g. for periodic systems it remains true.

Extension of the invariance principle to certain classes of systems that are e.g. asymptotically autonomous, or almost periodic, has been a popular research field. For such systems some weaker form of invariance, known as pseudo-invariance, can be identified [7].

The results above arise mainly from a restriction of the class of vector fields in order to deduce asymptotic stability by exploiting some form of invariance. In this paper we are trying to relax the conditions imposed on the vectorfield but set extra conditons on the derivative that guarantee asymptotic stability. We are in fact for nonautonomous systems pursuing a 'direct' approach to asymptotic stability, closer in spirit to the theorem of Barbashin and Krasovskii for autonomous systems - not as a corollary from a general statement akin to LaSalle's invariance principle. Explicitly: let $\dot{x}=f(x, t)$ be a nonautonomous differential equation and $V(x)$ a positive definite continuously differentiable functional with $\dot{V}(x, t) \leq 0$. The case $V(x, t)$ where $V$ depends explicitly on time will be examined in a forthcoming paper. The problem is to find weak extra conditions on $\dot{V}$ leading to asymptotic stability.

In Section 2 we state and prove our main result. In Section 3 the conditions of the main theorem are reformulated in integral form. This section is concluded with a short discussion where the theorem is compared with other stability results from the literature. In Section 4 we present a particular stability result for a class of linear nonautonomous systems. It shows that for some special classes the conditions of the main theorem may be weakened. We conclude the paper with an example for which we announce some new stability results. In forthcoming papers we will apply the theory to particular classes of systems in order to further illustrate the approach.

\section{An asymptotic stability criterion}

Theorem 1. Let $\mathcal{X}$ be an open subset of $\mathbf{R}^{n}$. Consider the differential equation

$$
\dot{x}=f(x, t)
$$

where

$$
f: \mathcal{X} \times \mathbf{R} \rightarrow \mathbf{R}^{n} .
$$

Assume existence and uniqueness of solutions. The following assumptions hold:

1. $f$ is measurable in $t$ for fixed $x$ and continuous in $x$ for fixed $t$.

2. $0 \in \mathcal{X}, f(0, t)=0 \forall t$. 
3. $f$ is locally Lipschitz in $x$ with bounded local Lipschitz function $l_{x}(t)$ in the neighborhood of $x$.

Let $V: \mathcal{U} \subset \mathcal{X} \rightarrow \mathbf{R}$ be a continuously differentiable map, for which there is some neighborhood $\mathcal{O} \subset \mathcal{U}$ of 0 such that the following properties hold:

Property 1. $V$ is positive definite:

$$
V(0)=0 \quad \text { and } \quad V(x)>0 \quad \text { for each } x \in \mathcal{O}, x \neq 0
$$

Property 2. $\forall x \in \mathcal{O}, t \in \mathbf{R}$ :

$$
\dot{V}(x, t):=\nabla V(x) . f(x, t) \leq 0
$$

Property 3. For each $q \in \mathcal{O},(q \neq 0), \exists$ a finite $s(q)>0$, such that for no sequence $\left(t_{j}\right)_{j \in \mathbf{N}} \rightarrow \infty$, when $j \rightarrow \infty$ (denoted as $\left.t_{j} \rightarrow \infty\right)$, the following is true:

$$
\lim _{t_{j} \rightarrow \infty} V\left(x\left(t_{j}+s(q) ; q, t_{j}\right)\right)=V(q) .
$$

Then (1) is asymptotically stable at the origin.

The formula $x\left(t_{j}+s(q) ; q, t_{j}\right)$ is standard notation for the state at time $t_{j}+s(q)$ corresponding to the solution of (1) with initial condition $q$ at time $t_{j}$. [8]:

For completeness we recall the definitions of stability and asymptotic stability

- stability; the system (1) is stable at the origin if for any $\varepsilon>0$ and for any $t_{0}$, there exists a $\delta\left(\varepsilon, t_{0}\right)>0$ such that $\left|x_{0}\right|<\delta$ implies $\left|x\left(t ; x_{0}, t_{0}\right)\right|<\varepsilon, t \geq t_{0}$

- attractivity; the system (1) is attractive at the origin if for each $t_{0}$ there exists a neighborhood of the origin such that for any $x_{0}$ in this neighborhood the following holds: $\lim _{t \rightarrow \infty} x\left(t ; x_{0}, t_{0}\right)=0$

- asymptotic stability; the system (1) is asymptotically stable at the origin if it is stable and attractive.

Proof. Take an open ball $B_{\varepsilon}(0)$ with radius $\varepsilon$ and centered at the origin, small enough such that $\bar{B}_{\varepsilon}(0)$ is included in $\mathcal{O}$.

Let $\alpha$ be the minimum value of $V$ on the sphere $S_{\varepsilon}(0)$. The number $\alpha$ exists since $V$ is continuous on $S_{\varepsilon}(0) ; \alpha>0$ by Property 1 .

Let $\mathcal{U}_{1}:=\{x: x \in \mathcal{O}$ and $V(x)<\alpha\}$. Then $0 \in \mathcal{U}_{1}$ and $\mathcal{U}_{1}$ is open. Consider an open ball $B_{\delta}(0) \subset \mathcal{U}_{1}$. Then with $x_{0} \in B_{\delta}(0), t_{0}$ arbitrary, the state $x\left(t ; x_{0}, t_{0}\right)$ belongs to $B_{\varepsilon}(0)$ for $t \geq t_{0}$ by Property 2 . Therefore the origin is stable.

Since the system is stable, there always exists a ball $B_{\delta_{1}}(0)$ such that any trajectory $x\left(t ; x_{0}, t_{0}\right), x_{0} \in B_{\delta_{1}}(0), t \geq t_{0}$ and $t_{0}$ arbitrary, stays inside $B_{\delta / 2}(0) \subset$ $B_{\delta}(0)$. For such a trajectory, by compactness of $\bar{B}_{\delta}(0)$ there exists an increasing sequence of times $t_{i}>t_{0}$, with $t_{i} \rightarrow \infty$ as $i \rightarrow \infty$ such that

$$
\lim _{t_{i} \rightarrow \infty} x\left(t_{i} ; x_{0}, t_{0}\right)=p
$$

with $p \in B_{\delta}(0)$. Assume that $p \neq 0$. 
Let $x_{i}$ denote $x\left(t_{i} ; x_{0}, t_{0}\right)$ with $x_{0} \in B_{\delta_{1}}(0)$. Consider the sequence $x\left(t_{i}+\right.$ $\left.s(p) ; x_{0}, t_{0}\right), t_{i} \rightarrow \infty$, with the sequence $t_{i}$ considered above and with $s(p)$ associated with $p$ according to Property 3. By continuity of solutions of differential equations with respect to initial conditions (cfr. assumption 3 on $f$ ), there exists $K>0$, such that:

$$
\left|x\left(t_{i}+s(p) ; x_{i}, t_{i}\right)-x\left(t_{i}+s(p) ; p, t_{i}\right)\right| \leq\left|x_{i}-p\right| e^{K . s(p)}
$$

Indeed, all solutions of (1) with initial condition $x_{0} \in B_{\delta_{1}}(0)$ remain in the compact set $\bar{B}_{\varepsilon}(0)$ and by assumption 3 on the differential equation (1) the constant $\mathrm{K}$ can be taken to be the maximum of the bounded Lipschitz functions $l_{x}(t)$ associated with a finite covering of $\bar{B}_{\varepsilon}(0)$. Then

$$
\lim _{i \rightarrow \infty}\left|x\left(t_{i}+s(p) ; x_{i}, t_{i}\right)-x\left(t_{i}+s(p) ; p, t_{i}\right)\right|=0
$$

since $\lim _{i \rightarrow \infty} x_{i}=p$. Since both $x\left(t_{i}+s(p) ; x_{i}, t_{i}\right)$ and $x\left(t_{i}+s(p) ; p, t_{i}\right)$ belong to $B_{\varepsilon}(0)$ for al $i$ and since $V$ is continuous and therefore uniformly continuous on $\bar{B}_{\varepsilon}(0)$, (2) implies that

$$
\lim _{i \rightarrow \infty}\left|V\left(x\left(t_{i}+s(p) ; x_{i}, t_{i}\right)\right)-V\left(x\left(t_{i}+s(p) ; p, t_{i}\right)\right)\right|=0 .
$$

Consider the sequence of elements $V\left(x\left(t_{i}+s(p) ; p, t_{i}\right)\right)$ with $t_{i} \rightarrow \infty$. These elements belong to the interval $[0, V(p)]$ by Property 1 and Property 2 . Then there exists a subsequence $t_{i_{\text {sub }}} \rightarrow \infty$ of the sequence $\left(t_{i}\right)$ corresponding to a subsequence $i_{s u b} \rightarrow \infty$ such that

$$
\lim _{t_{i_{s u b}} \rightarrow \infty} V\left(x\left(t_{i_{\text {sub }}}+s(p) ; p, t_{i_{\text {sub }}}\right)\right)=a
$$

where $a<V(p)$ by Property 3 .

From the triangle inequality we obtain with $x_{i_{\text {sub }}}:=x\left(t_{i_{\text {sub }}} ; x_{0}, t_{0}\right)$

$$
\begin{aligned}
\mid V\left(x \left(t_{i_{s u b}}\right.\right. & \left.\left.+s(p) ; x_{i_{\text {sub }}}, t_{i_{\text {sub }}}\right)\right)-a \mid \\
& \leq\left|V\left(x\left(t_{i_{\text {sub }}}+s(p) ; x_{i_{\text {sub }}}, t_{i_{\text {sub }}}\right)\right)-V\left(x\left(t_{i_{\text {sub }}}+s(p) ; p, t_{i_{\text {sub }}}\right)\right)\right| \\
& +\left|V\left(x\left(t_{i_{\text {sub }}}+s(p) ; p, t_{i_{\text {sub }}}\right)\right)-a\right|
\end{aligned}
$$

and by (3) and (4) we obtain from (5)

$$
\lim _{i_{s u b} \rightarrow \infty} V\left(x\left(t_{i_{s u b}}+s(p) ; x_{i_{s u b}}, t_{i_{s u b}}\right)\right)=a<V(p) .
$$

On the other hand, since $\lim _{i \rightarrow \infty} x\left(t_{i} ; x_{0}, t_{0}\right)=p$ and since $V$ is continuous at $p$

$$
\lim _{i \rightarrow \infty} V\left(x\left(t_{i} ; x_{0}, t_{0}\right)\right)=V(p) .
$$

Since $V\left(x\left(t ; x_{0}, t_{0}\right)\right.$ is nonincreasing by Property 2 and $\geq 0$ by Property 1 , its limit exists and equals $V(p)$ by $(7)$. Therefore

$$
V\left(x\left(t ; x_{0}, t_{0}\right) \geq V(p), \quad \forall t .\right.
$$


But (6) states that $V\left(x\left(t_{i_{\text {sub }}}+s(p) ; x_{i_{\text {sub }}}, t_{i_{\text {sub }}}\right)\right)<V(p)$ for $t_{i_{\text {sub }}}$ large enough. This is in contradiction with (8) since all states

$$
x\left(t_{i_{s u b}}+s(p) ; x_{i_{s u b}}, t_{i_{s u b}}\right)
$$

belong to the trajectory $x\left(t ; x_{0}, t_{0}\right)$.

Therefore our original assumption that $p \neq 0$ is impossible. This proves that the only positive limit point of $x\left(t ; x_{0}, t_{0}\right)$ is the origin. Since the system is stable, asymptotic stability of the origin follows.

3. Reformulation of Property 3. The appeal of the direct method of Liapunov arises from the fact that the stability properties of (1) are examined without a knowledge of its solutions. Property 3 involves trajectories and so it seems that most of the appeal of Liapunov's approach has been lost. In the autonomous case however, the theorem of Barbashin and Krasovskii or more general LaSalle's invariance principle are extremely valuable contributions to the stability literature in spite of the fact that they also rely on information of particular trajectories - those restricted to the subset defined by $\{x: \dot{V}(x)=0\}$.

Property 3 is the crucial extra condition needed besides the negative semidefiniteness of $\dot{V}(x, t)$, in order to guarantee asymptotic stability of (1). It plays a role similar to the conditions on the trajectories one finds in the statements of the theorem of Barbashin and Krasovskii or LaSalle's invariance principle. These theorems suggest to formulate Property 3 in terms of $\dot{V}(x, t)$ which is easily done as follows.

With $p, s(p)$ and the sequence $\left(t_{i}\right)$ as above, the following holds:

$$
V\left(x\left(t_{i}+s(p) ; p, t_{i}\right)\right)-V\left(x\left(t_{i} ; p, t_{i}\right)\right)=\int_{0}^{s(p)} \frac{d V\left(x\left(t_{i}+\tau ; p, t_{i}\right)\right)}{d \tau} d \tau .
$$

With

$$
\int_{0}^{s(p)} \frac{d V\left(x\left(t_{i}+\tau ; p, t_{i}\right)\right)}{d \tau} d \tau=\int_{0}^{s(p)} \frac{d V\left(x\left(t_{i}+\tau ; p, t_{i}\right)\right)}{d x} \cdot \frac{d x\left(t_{i}+\tau ; p, t_{i}\right)}{d \tau} d \tau
$$

and since

$$
\frac{d x\left(t_{i}+\tau ; p, t_{i}\right)}{d \tau}=f\left(x\left(t_{i}+\tau ; p, t_{i}\right), t_{i}+\tau\right)
$$

and also since $V\left(x\left(t_{i} ; p, t_{i}\right)\right)=V(p),(9)$ is rewritten as

$$
V\left(x\left(t_{i}+s(p) ; p, t_{i}\right)\right)-V(p)=\int_{0}^{s(p)} \dot{V}\left(x\left(t_{i}+\tau ; p, t_{i}\right), t_{i}+\tau\right) d \tau .
$$

Property 3 is then immediately rephrased as follows: 
Property 4. For each $p \in \mathcal{O}$ there is a positive $s(p)$ such that for no sequence $t_{i} \rightarrow \infty$

$$
\lim _{t_{i} \rightarrow \infty} \int_{0}^{s(p)} \dot{V}\left(x\left(t_{i}+\tau ; p, t_{i}\right), t_{i}+\tau\right) d \tau=0 .
$$

By (10) and since $\dot{V} \leq 0$, Property 3 (or equivalently Property 4 ) states that for each initial state $p$ there exists a time interval $s(p)$ such that for no sequence of initial times $t_{i}$ tending to infinity, the $V$-values at $x\left(t_{i}+s(p) ; p, t_{i}\right)$ tend to the constant value $V(p)$. This implies - since $V$ is nonincreasing along trajectoriesthat $V$ does not tend to the constant $V(p)$ along intervals $\left[t_{i}, t_{i}+s(p)\right]$. Recall that in the case of autonomous systems asymptotic stability is guaranteed by the theorem of Barbashin and Krasovskii if $\dot{V} \leq 0$ and there are no trajectories leaving $V$ invariant, i.e. belonging to the set $\{x: \dot{V}(x)=0\}$. In this context Property 4 is then best interpreted as the preclusion of a type of invariance of $V$ so as to guarantee asymptotic stability. We would like to emphasize - as already mentioned in the Introduction - that we have not assumed any form of invariance on the limit sets of the flow itself.

4. Weaker form of the asymptotic stability property for linear timevariant systems. Consider the linear time-variant system

$$
\dot{x}=A(t) x .
$$

Assume there exists a positive definite quadratic form $W(x):=x^{T} P x$ such that

$$
\dot{W}(x, t)=x^{T}\left(A^{T}(t) P+P A(t)\right) x=: x^{T} Q(t) x \leq 0 .
$$

Then for any solution $x\left(t_{0}+s ; x_{0}, t_{0}\right)$ of (11) the following is true: $W\left(x\left(t_{0}+\right.\right.$ $\left.\left.s ; x_{0}, t_{0}\right)\right) \leq W\left(x_{0}\right)$.

Consider now the solutions of (11) $x\left(t_{i}+s ; x_{i}, t_{i}\right)$ and $x\left(t_{i}+s ; p, t_{i}\right)$ with $x_{i}$ and $t_{i}$ defined above, then because of linearity of (11) their difference also satisfies (11) and therefore

$$
W\left(x\left(t_{i}+s ; x_{i}, t_{i}\right)-x\left(t_{i}+s ; p, t_{i}\right)\right) \leq W\left(x_{i}-p\right) .
$$

With $i \rightarrow \infty$ (i.e. when $t_{i} \rightarrow \infty$ and $x_{i} \rightarrow p$ ) the right hand side tends to zero. Since it is independent of $s$ this relation implies that

$$
\mid\left(x\left(t_{i}+s ; x_{i}, t_{i}\right)-x\left(t_{i}+s ; p, t_{i}\right) \mid \rightarrow 0\right.
$$

uniformly in $s$ as functions defined on $[0, \infty)$.

Let $\left(x_{i}, t_{i}\right)$ be defined as in Section 3 (i.e. belonging to the solution $x\left(t_{0}+\right.$ $\left.s ; x_{0}, t_{0}\right)$ and such that $x_{i} \rightarrow p$, for $\left.i \rightarrow \infty\right)$ then by stability

$$
\left.x^{T}\left(t_{i}+s ; p, t_{i}\right) P x\left(t_{i}+s ; p, t_{i}\right)\right) \leq p^{T} P p \quad \forall s
$$

and also

$$
x^{T}\left(t_{i}+s ; x_{i}, t_{i}\right) P x\left(t_{i}+s ; x_{i}, t_{i}\right) \leq x_{i}^{T} P x_{i} \leq x_{0}^{T} P x_{0} \quad \forall s .
$$


In other words there exists a compact ball $B$ in $\mathbf{R}^{n}$ that contains all points $x\left(t_{i}+s ; p, t_{i}\right)$ and $x\left(t_{i}+s ; x_{i}, t_{i}\right)$ with $s \geq 0$. Since $W$ is uniformly continuous on $B$, it follows that

$$
W\left(x\left(t_{i}+s ; x_{i}, t_{i}\right)\right)-W\left(x\left(t_{i}+s ; p, t_{i}\right)\right) \rightarrow 0
$$

uniformly in $s$ as functions defined on $[0, \infty)$ when $t_{i} \rightarrow \infty$.

Indeed, given $\varepsilon$, by uniform continuity of $W$ there exists a number $\delta$ such that

$$
\left|W\left(x\left(t_{i}+s ; x_{i}, t_{i}\right)\right)-W\left(x\left(t_{i}+s ; p, t_{i}\right)\right)\right|<\varepsilon
$$

if

$$
\left|\left(x\left(t_{i}+s ; x_{i}, t_{i}\right)\right)-\left(x\left(t_{i}+s ; p, t_{i}\right)\right)\right|<\delta
$$

the latter being satisfied for $t_{i}$ larger than some number $N$ by uniform convergence to zero of

$$
\left(x\left(t_{i}+s ; x_{i}, t_{i}\right)\right)-\left(x\left(t_{i}+s ; p, t_{i}\right)\right)
$$

when $t_{i} \rightarrow \infty$ (cf. (12)). Since

$$
-\varepsilon<W\left(x\left(t_{i}+s ; x_{i}, t_{i}\right)\right)-W\left(x\left(t_{i}+s ; p, t_{i}\right)\right)<\varepsilon
$$

for $t_{i}>N$ and since $W$ is nonincreasing, the limit of the middle term, with $t_{i}$ fixed, exists for $s \rightarrow \infty$ (and is equal to the difference of the limits of both terms). Therefore

$$
-\varepsilon \leq \lim _{s \rightarrow \infty} W\left(x\left(t_{i}+s ; x_{i}, t_{i}\right)\right)-\lim _{s \rightarrow \infty} W\left(x\left(t_{i}+s ; p, t_{i}\right)\right) \leq \varepsilon
$$

for $t_{i}>N$, i.e.

$$
\left|\lim _{s \rightarrow \infty} W\left(x\left(t_{i}+s ; x_{i}, t_{i}\right)\right)-\lim _{s \rightarrow \infty} W\left(x\left(t_{i}+s ; p, t_{i}\right)\right)\right| \rightarrow 0 \text { for } t_{i} \rightarrow \infty .
$$

On the other hand - assuming Properties 1, 2, 3- the following is true (see Section 2):

$$
\lim _{s \rightarrow \infty} W\left(x\left(t_{i_{s u b}}+s ; p, t_{i_{s u b}}\right)\right) \rightarrow a \quad \text { when } t_{i_{s u b}} \rightarrow \infty
$$

with $a<W(p)$ as above.

From (14) and (15) we obtain by the triangle inequality

$$
\lim _{i_{s u b} \rightarrow \infty}\left(\lim _{s \rightarrow \infty} W\left(x\left(t_{i_{s u b}}+s ; x_{i_{s u b}}, t_{i_{s u b}}\right)\right)\right)=a<V(p) .
$$

This leads to a contradiction as shown in Section 2. We obtain the following theorem:

Theorem 2. Consider the linear system $\dot{x}=A(t) x$. Assume there exists a positive definite matrix $P$ such that $W=x^{T} P x$ has negative semi-definite derivative. If $A(t)$ is measurable and bounded and there is no sequence $t_{i} \rightarrow \infty$ such that (with $p \neq 0)$

$$
\int_{0}^{\infty} \dot{W}\left(x\left(t_{i}+s ; p, t_{i}\right)\right) d s \rightarrow 0 \quad \text { for } t_{i} \rightarrow \infty
$$

then $\dot{x}=A(t) x$ is asymptotically stable. 
EXAMPLE. We announce the following theorem which is derived based on Theorem 2 above. For details one is referred to a forthcoming paper [1]. Consider the following time-variant differential equation

$$
\dot{x}=-m(t) m(t)^{T} x
$$

with $x(t)$ and $m(t) n$-dimensional. This equation or related versions arise in identification and control problems and have been studied extensively ([2], [5]). Usually $x(t)$ represents the parameter error that is driven to zero, based on an observation of the error $m^{T}(t) x(t)$.

A set of stability results is related to the notion of persistency of excitation of $m(t)$. Let $m(t)$ be a regulated function. It is called persistently exciting if there exists $T>0$ such that $\forall s$

$$
\alpha I \leq \int_{s}^{s+T} m(t) m(t)^{T} d t \leq \beta I
$$

with $\alpha>0$ and $\beta>0$. It has been shown ([5], [2]) that this is a necessary and sufficient condition for exponential stability, (trajectories are bounded above and below by exponentials). The importance of this result is that it accomodates a wide class of signals, beyond the (almost) periodicity constraint of Lasalle's principle, still securing asymptotic stability. We now present the following (weaker) sufficient condition for asymptotic stability.

THEOREM 3. Let $m(t)$ be regulated and bounded. A sufficient condition for asymptotic stability of (16) is that for each $t$ there exists a $T(t)>t$ such that

$$
\alpha I \leq \int_{t}^{T(t)} m(\tau) m(\tau)^{T} d \tau \leq \beta I
$$

with $\beta \geq \alpha>0$.

Theorem 3 represents a generalization of the stability results concerning (16) featuring in $[2,5]$. Indeed the sufficient condition is relaxed in that the integration interval $T(t)-t$ is no longer constant. As an example consider the differential equation $\dot{x}=-(1 / t) x$ for $t \geq 1$. This differential equation is asymptotically stable as can be verified by direct computation. This follows also from (18) for $T(t)=2 t$ but the equation does not satisfy (17)

Acknowledgment. During the course of this work the author has benefited from several discussions with R. Sepulchre and J. L. Willems.

\section{References}

[1] D. Aeyels and R. Sepulchre, On the convergence of a time-variant linear differential equation arising in identification, to appear in Kybernetika. 
[2] B. D. O. Anderson, Exponential stability of linear equations arising in adaptive identification, IEEE Trans. Automat. Control 22 (1977), 84-88.

[3] E. A. Barbashin and N. N. Krasovskii, Stability of motion in the large, Dokl. Akad. Nauk SSSR 86 (1952), 453-456.

[4] H. K. Khalil, Nonlinear Systems, Macmillan Publishing Company, New York, 1992.

[5] G. Kreisselmeier, Adaptive observers with exponential rate of convergence, Trans. Automat. Control 22 (1977), 2-8.

[6] J. P. LaSalle, Stability of nonautonomous systems, Nonlinear Anal. 1 (1976), 83-91.

[7] N. Rouche, P. Habets and M. Laloy, Stability Theory by Liapunov's Direct Method, Springer-Verlag, New York, 1977.

[8] J. L. Willems, Stability Theory of Dynamical Systems, Nelson, London, 1970. 